\title{
NON-INVASIVE ASSESSMENT OF LIVER FIBROSIS USING LIVER ELASTOGRAPHY (FIBROSCAN) IN PATIENTS WITH CHRONIC INFECTION HBV AND HCV
}

\author{
Andreea Rădășan², Mihai Voiculescu ${ }^{1,2}$, Laura Elena Iliescu ${ }^{1,2}$ \\ 1 "Carol Davila" University of Medicine and Pharmacy, Bucharest, Romania \\ ${ }^{2}$ Internal Medicine Department, Fundeni Clinical Institute, Bucharest, Romania
}

\begin{abstract}
Introduction. In recent years there have been major advances in the treatment and prevention of viral hepatitis, but this pathology is still a major health and socio-economic problem. The defining element for this disease is the liver fibrosis, a histological component of particular importance due to its role in the formation of liver lesions of cirrhosis. Thus, an essential step in the management of chronic viral hepatitis is the detection and measurement of liver fibrosis. Today we have invasive methods for detecting liver fibrosis, the liver puncture biopsy, and non-invasive methods, which in turn are divided into serum methods and imaging methods (2).

Purpose of the Study. The aim of this study is to determine if transcutaneous elastography (FibroScan) is equally reliable in case of viral hepatitis $B$, as with viral hepatitis $C$.

Material and Method: The study comprises a total of 1,127 patients with liver disease of HBV and HCV aetiology. These patients were examined using FibroScan in the period July 2009 - April 2011.

Results: Of the 1,177 patients investigated using FibroScan, 40 underwent liver biopsy as well. Of these, $82 \%$ have obtained same stages of liver fibrosis in these two investigations, and $18 \%$ achieved different stages of fibrosis in these two tests. For the patients with HCV infection, we obtained a $67.81 \%$ match of the two non-invasive tests, FibroScan and FibroMax, and a $79.16 \%$ match of FibroScan compared to the Liver Biopsy. For the $B$ virus, the compliance is even better, of $75 \%$ (FibroScan - FibroMax), and $80 \%$ for FibroScan - LB.

Conclusions: FibroScan is one of the non-invasive assessment methods of liver fibrosis with diagnose accuracy similar to FibroMax and close to Liver Biopsy
\end{abstract}

Keywords: Hepatitis B, Hepatitis C, FibroScan, FibroMax, Fibrosis, accuracy, match.

\section{INTRODUCTION}

Liver fibrosis plays a key role in the formation of liver lesions of liver cirrhosis. Fibrosis can also be seen in other liver diseases, not only in cirrhosis, but it is an essential component for this condition, distinctive for this illness. The immediate consequences of the fibrosis are the disorganization of the liver structure and the replacement of the functional liver tissue with amorphous, non-functional tissue. Screening and assessing liver fibrosis represents an important milestone in the diagnosis of liver disease. Recent studies show that liver fibrosis, at least until a certain stage of evolution, can be stopped and eventually its regression could be achieved by removing the cause and stopping the fibrogenesis. Under these conditions it is imperative to benefit from one or more methods of monitoring liver fibrosis (2).

There are several types of screening methods for fibrosis. The methods for early detection of 
liver fibrosis are invasive, represented by liver puncture biopsy and non-invasive methods. Non-invasive methods are also of several types: serum markers (direct and indirect) and imaging methods. Direct markers are rarely used in clinical trials, and are typically used in the fundamental research. Over time there has been a competition between the invasive and non-invasive methods for diagnosing liver fibrosis. The main advantage of the invasive methods, represented by liver puncture biopsy, is that it offers the possibility of assessing how the fibrosis it is distributed in the context of liver architecture. Moreover, the liver biopsy offers the advantage of diagnosing the related lesions, such as fat loading, iron deposits, necrosis, etc. Biopsy has its disadvantages, of which the most important are the traumatic character and representativeness of the fragment, taking into account that the best fragment is no more than $1 / 50,000$ of the liver mass. Non-invasive tests are cheaper and, as suggested by their name, are atraumatic and can be repeated whenever needed. But their accuracy is low in some cases, so we need a "consortium" of indirect tests for a true image on the liver fibrosis. Out of these tests, FibroScan has established itself as an imaging method with good diagnostic accuracy reported to the liver biopsy, considered the "gold-standard" of liver lesion assessment (1-3).

\section{PURPOSE}

Given that more and more patients refuse liver biopsy, preferring a non-invasive method, the purpose of this study was to determine the accuracy of liver fibrosis assessment using the transcutaneous elastography in patients with chronic HBV infection. In Romania, chronic HBV infection represents a health problem, at least as important as that of HCV chronic hepatitis, despite the existence of a method of prevention. The patient wants a method for assessing liver fibrosis, primarily non-invasive, and then at a low cost and high reproducibility.
The main objectives of the study are:

1. Testing the accuracy of FibroScan results in assessing liver fibrosis in patients with chronic HBV.

2. Comparing FibroScan results with liver biopsy results and those achieved with FibroMax and trying to demonstrate which is the best non-invasive method for liver fibrosis evaluation.

3. Comparing FibroScan results in patients with chronic HBV infection with those of HCV patients.

\section{MATERIALS AND METHODS}

The study carried out is prospective. The data necessary for the study were taken from the observation chart of patients hospitalized in the Internal Medicine and Nephrology Department of Fundeni Clinical Institute, between July 2009 and April 2011. A number of 1,127 patients with HBV and HCV viral infection were included. They were hospitalized in Fundeni Clinic of Internal Medicine and Nephrology in the period July 2009 - April 2011. The inclusion criteria are represented by patients with viral liver disease (HBV, HCV) proven by virology tests and patients with chronic viral hepatitis with and without antiviral treatment. The exclusion criteria are represented by patients with ascites, pregnancy, cardiac stimulation devices and obesity.

The subjects were examined using the FibroScan No. 502 developed by Echosens (Paris, France). Data were entered into a single database MS Access 2010. MS Excel 2010 and IBM SPSS Statistics 19 were used for the graphics and statistical calculations.

\section{RESULTS}

All patients were examined using FibroScan in the period July 2009 - April 2011. Each patient underwent at least one FibroScan examination, so the total number of elastography tests of 
these patients is 3,750. The patients' average age was 52.5 years. Of these 1,127 patients, 501 (44.45\%) were men and 626 (55.54\%) were women, with approximately equal gender distribution.

Of the total 1,127 patients, 351 (31.14\%) were diagnosed with chronic HBV infection, with a slight predominance in women (58.97\%) than men (41.02\%) (Fig. 1). Of these, 339 patients $(96.58 \%)$ were examined with FibroScan. For a rate of $3.41 \%$, the examiner could not obtain a result due to technical reasons: obesity, severe abdominal meteorism, etc. Only 72 patients $(20.51 \%)$ underwent the FibroMax test.

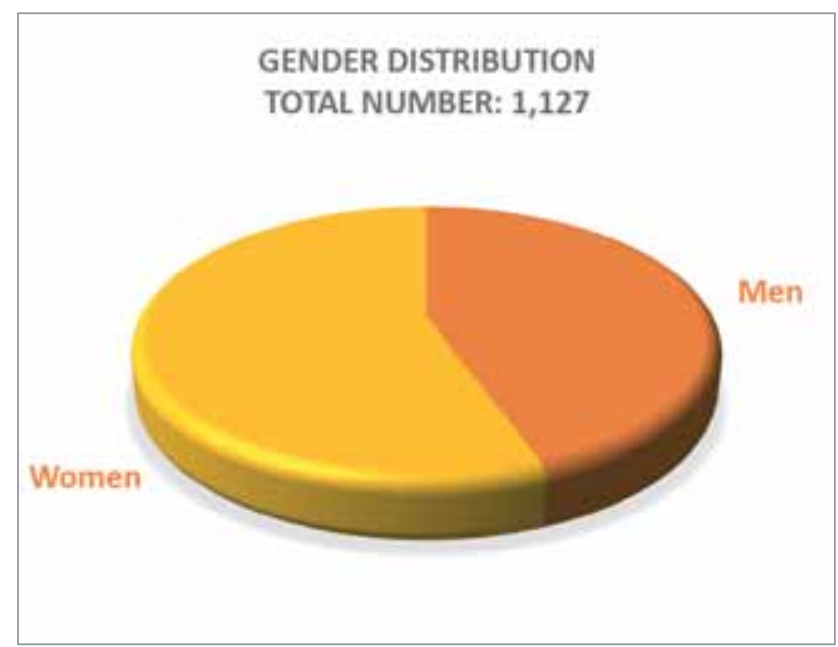

Figure 1. Gender distribution in patients

The majority of patients had F0 and F1 fibrosis ( $\mathrm{F} 0=35.89 \%$, F1 = 36.75\%) (Fig. 2). Average fibrosis was found in a much smaller percentage, F2 (10.82\%) and very few had advanced degrees of fibrosis $(\mathrm{F} 3=6.55 \%=6.55 \% \mathrm{~F} 4)$ (Fig. 2).

The comparison was made with 571 patients with chronic HCV (50.66\%), the predominance of women was also noted, 65.84\% (376 patients). Of the 571 patients with chronic hepatitis C, 15 subjects $(2.62 \%)$ were unable to achieve a FibroScan result due to the same technical reasons outlined above (obesity, severe abdominal meteorism). Unlike patients with HBV infection, roughly equal percentages were obtained in subjects diagnosed with chronic hepatitis HCV

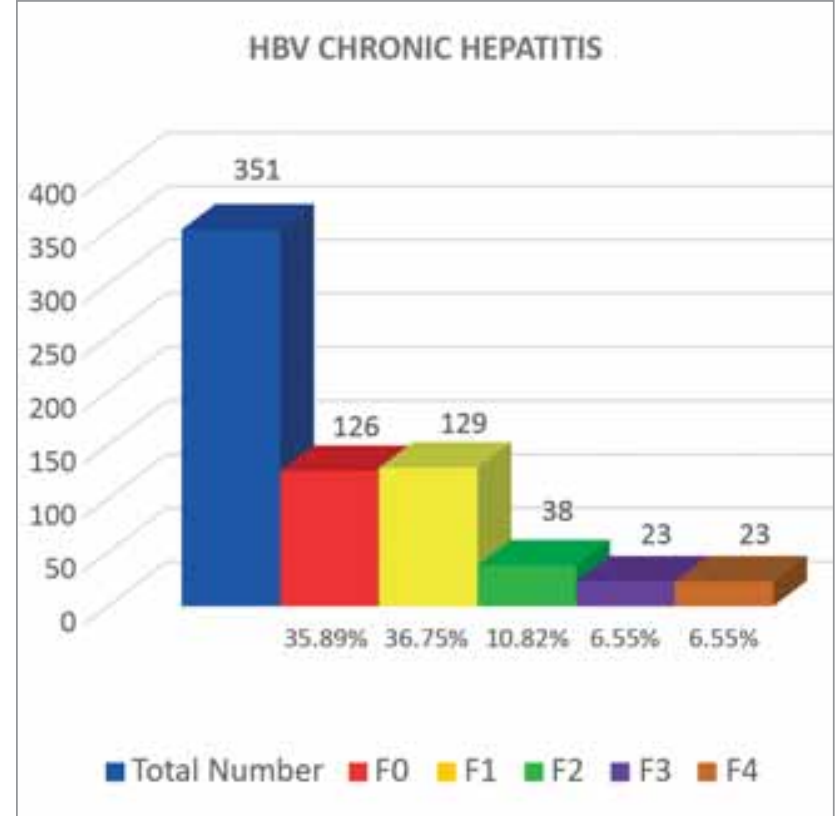

Figure 2. The degrees of hepatic fibrosis in patients with chronic HBV

in fibrosis ranges F0 (19.26\%), F1 (28.54\%) and F2 (19.08\%), with a slight predominance of average fibrosis, F2. However, an advanced fibrosis, F3 and F4 was identified in a higher percentage than in patients with chronic HBV (F3 and F4 $=14.18 \%=16.63 \%$ ) (Fig. 3 ).

Of the 1,127 subjects, 55 patients (4.88\%) were diagnosed with HBV liver cirrhosis. Of these, 60\% (33 subjects) were men and 40\% (22 subjects) were women. A total of 3 patients

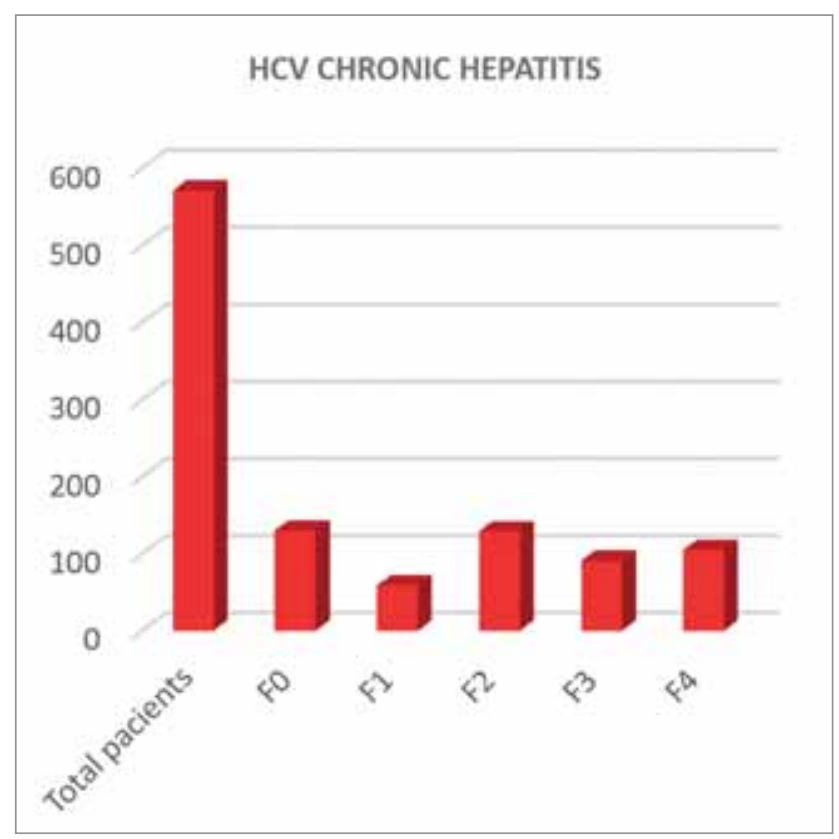

Figure 3. The degrees of hepatic fibrosis in patients with chronic $\mathrm{HCV}$ 
could not get a result in the FibroScan examination due to obesity, abdominal meteorism and ascites fluid. Of the patients diagnosed with HBV liver cirrhosis, a rate of $65,45 \%$ had F4 liver fibrosis values, on FibroScan examination. A percentage of $18.18 \%$ achieved METAVIR F3 fibrosis score at and no patient in the F0 range (Fig. 4). Seven patients chose to perform both tests, FibroScan and FibroMax.

Regarding HCV liver cirrhosis, the number of patients investigated was much higher, 154 patients (13.66\%). Of these, 88 subjects (57.14\%) were women and 66 subjects (42.85\%) are men. 370 FibroScan examinations have been performed in these patients. Only 5 patients had difficulties in obtaining a FibroScan result, and 21 of the 154 have chosen to perform both tests, FibroMax and FibroScan. A rate of $84.41 \%$ F4 fibrosis score was diagnosed in patients with $\mathrm{HCV}$ liver cirrhosis, and no patient fell in the F0 range (Fig. 5).

FibroScan and FibroMax are the main noninvasive tests for the assessment of liver fibrosis currently available. FibroScan was initially validated for the infection with hepatitis $C$ virus, and has been included recently in the diagnosis and treatment guide of chronic HBV infection, and FibroMax is validated for all chronic viral hepatitis. Patients in this study were evaluated using FibroScan (1,127 patients) and FibroMax (187 patients). As for the HBV chronic hepatitis, a $75 \%$ consistency of two tests was obtained (similar degrees of fibrosis). A difference of only one degree was obtained in 9 patients, and a difference of 2 or more degrees of fibrosis was obtained in 8 patients. Of the 8 patients, 5 had previously performed the FibroMax test 1-2 years prior to the FibroScan examination (8-10).

A $67.81 \%$ match was obtained for HCV chronic hepatitis. A difference of 2 or more degrees was achieved in 14 patients.

For the HBV liver cirrhosis, the match obtained was of $71.42 \%$. Two patients achieved a higher value in FibroScan compared to FibroMax, but had undergone FibroMax 1-2 years

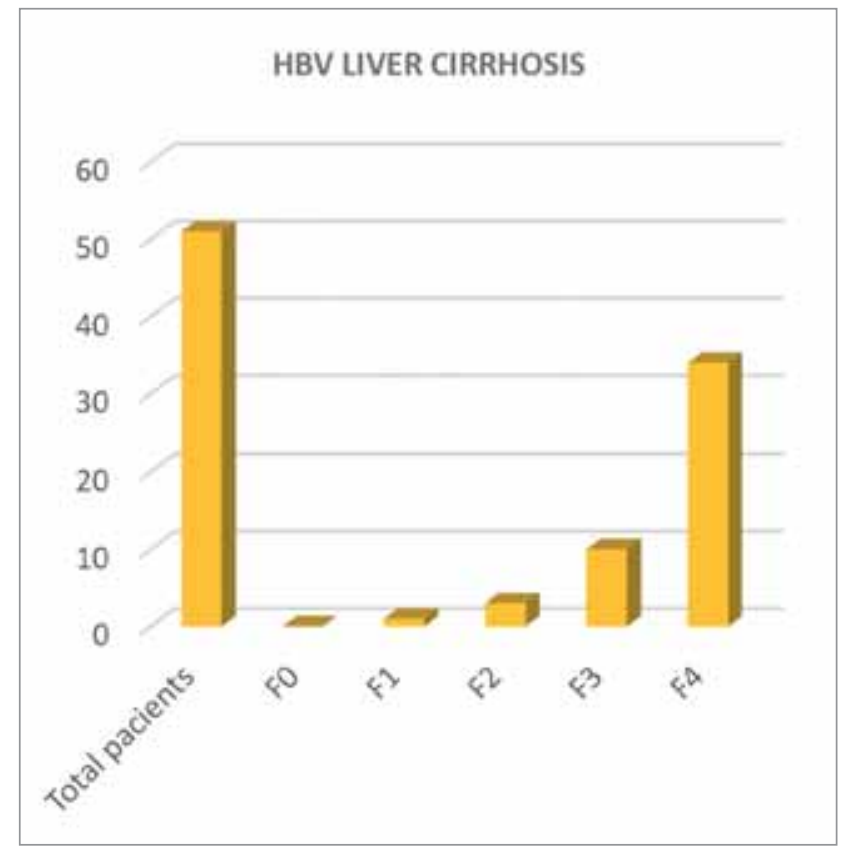

Figure 4. The degrees of hepatic fibrosis in patients with HBV liver cirrhosis

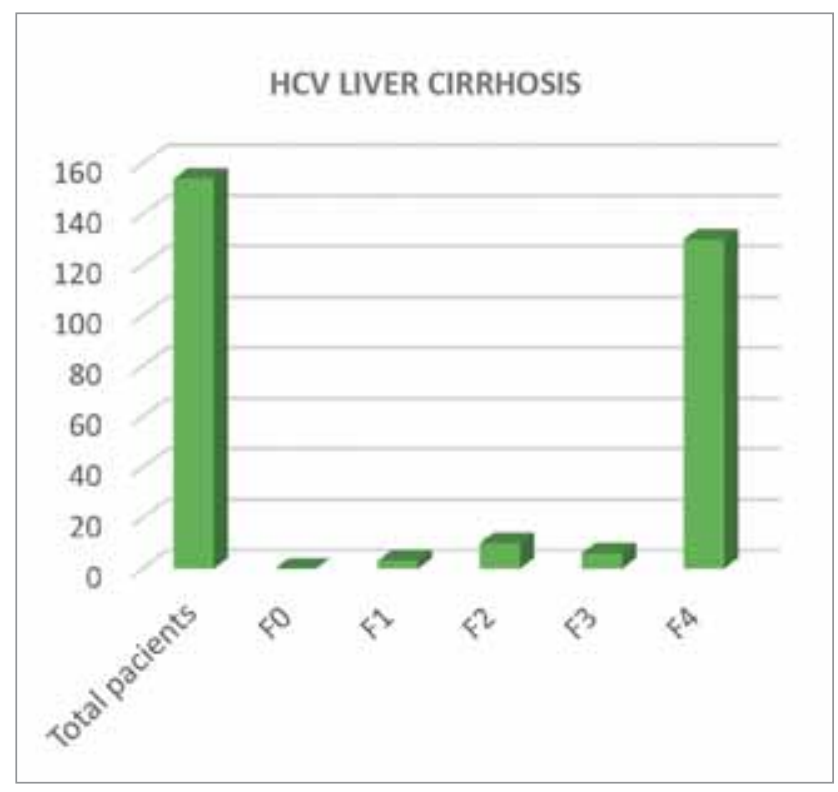

Figure 5. The degrees of hepatic fibrosis in patients with HCV liver cirrhosis

prior to elastography examination. The match in HCV cirrhotic hepatitis was 66.66\%.

Compared to patients with hepatitis $\mathrm{C}$ virus, a $67.81 \%$ match for of the two non-invasive tests FibroScan and FibroMax was obtained, and a $79.16 \%$ match of FibroScan compared to Liver Biopsy (Fig. 6). For B virus, the match is even better than $75 \%$ (FibroScan - FibroMax), i.e. $80 \%$ for FibroScan - LB (Fig. 7). 


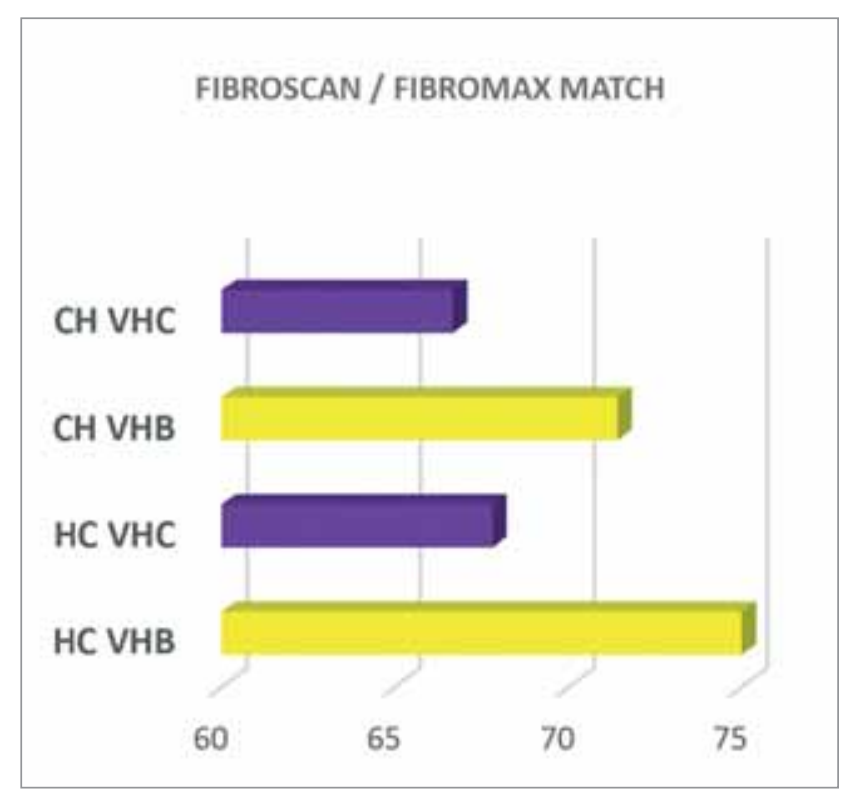

Figure 6. The match of the two non-invasive assessment types of liver fibrosis

\section{CONCLUSIONS}

1. FibroScan is one of the non-invasive assessment method for liver fibrosis with diagnose accuracy similar to FibroMax and close to Liver Biopsy.

2. In HCV chronic hepatitis, FibroScan assesses liver fibrosis with $67.81 \%$ accuracy compared to FibroMax and $79.16 \%$ compared to

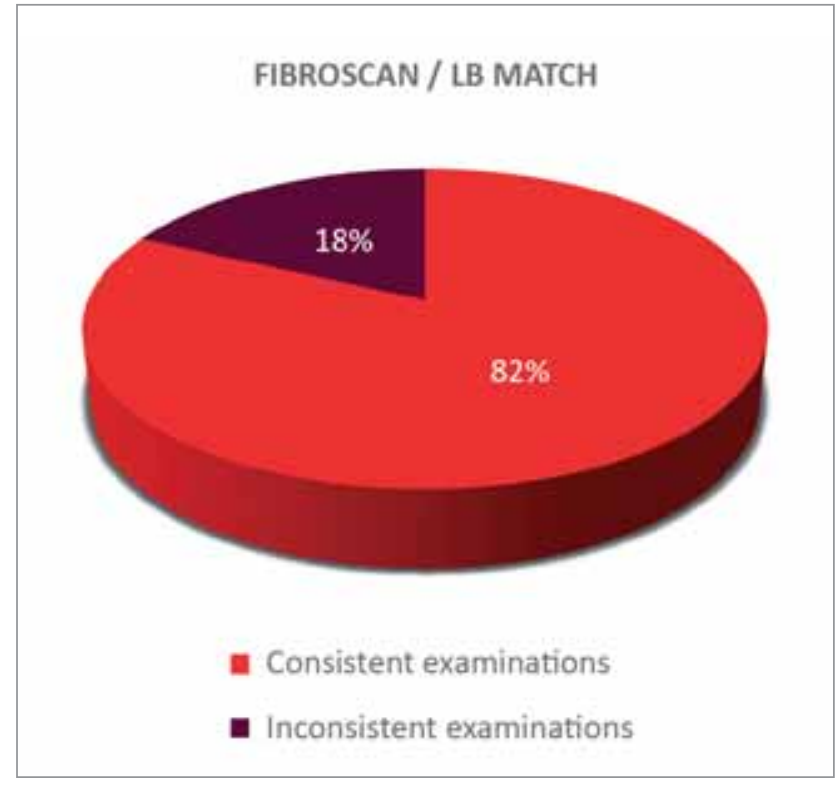

Figure 7. FibroScan - Liver Biopsy Match

liver puncture biopsy, and the replacement of liver puncture biopsy can be possible in the future in this category of patients.

3. In HBV chronic hepatitis, FibroScan assesses with $75 \%$ accuracy compared to FibroMax, and $80 \%$ compared to liver puncture biopsy.

4. In the study we conducted, FibroScan proved to be a simple, reproducible method with a very low rate of errors.

\section{REFERENCES}

1. Mihai Voiculescu, lliescu L et al. A cross sectional Eipdemiological Study of HBV, HCV, HDV and HEV Prevalence in the SubCarpathian and South-Eastern Regions of Romania. J Gastrointestin Liver Dis 2010; 43-48.

2. Mihaila Gabriel. Fibroza Hepatica, Ed. Teora, 1998

3. Deca C., Deac M. Puncţia Biopsie Hepatică: Modalitate de evaluare a fibrozei hepatice în hepatita cronică virală C; Acta Medica Transilvania; 2(264-66), 2010

4. Molnar G.B., Popa S., Jebeleanu C., Damian C. Studiul prevalentei markerilor serici ai infecţiei cu virusurile hepatitelor în anamneza epidemiologică a populaţiei. Bacteriol Virusol Parzitol Epidemiol 1994; 30:141-150.

5. Gheorghe L., lacob S., Csiki I.E. Prevalence of hepatitis C in Romania: different from European rates? J Hepatol 2008; 49: 661-662.

6. Deac M. Actualităţi în fibrogeneza hepatică. Sibiul Medical 6, 1, 1995; $14-17$.

7. Yen Ngo, Yves Benhamou. An accurate definition of the status of inactive hepatitis $B$ virus carrier by a combinations of biomarkers (FibroTest-ActiTest) and viral load, Plos One 3(7), July 2008.

8. Marianne Ziol et al. Noninvasive Assessment of Liver Fibrosis by Measurment of Stiffness in patients with Chronic Hepatits C; Hepatology 2005, 41: 48-45.

9. Bedossa P. Comment classer une hepatite chronique. Anne Pathol 1995; 15: 316-318

10. Mirella Fraquelli, Massimo Colombo. Reproductibility of transient elastogrphy in the evaluation of liver fibrosis in patients with chronic liver disease; Gut 2007; 56: 968-973.

11. Laurent Castera et al. Non-invasive evaluation of liver fibrosis using transient elastography; Journal of Hepatology 48 (2008) 835-847

12. Sebastiani G., Alberti A. Non-invasive fibrosis biomarkers reduce but not substitute the need for liver biopsy. World J Gastroenterology 2006; 12:3682-3694. 